\title{
A tough transition
}

\section{What is holding back biotechnology in the developing world? Peter A. Singer and his colleagues listen to those on the ground.}

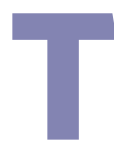

he path from basic scientific discovery to effective therapy is rarely rapid or simple, especially in the developing world. Making this transition easier is a sizeable and pressing problem. What is the best way to tackle such a complex issue?

One important step is to identify the factors that help and hinder the uptake of health-related biotechnology in developing countries. Although knowledge about these factors in developing regions is lacking ${ }^{1}$, the spread of technologies and ideas across cultures has been studied extensively for several decades. The complex issues involved in the development of new technologies cover areas as diverse as science-capacity building ${ }^{2}$, culture $^{3}$, economic analysis ${ }^{4}$, foreign investment and imports ${ }^{5}$, public-private product-development partnerships ${ }^{6}$, intellectual property ${ }^{7}$ and political policy ${ }^{8}$. These issues, however, have mostly been explored in the context of the developed world and in isolation from one another, which means that the bigger picture remains unclear.

The diversity of factors involved in the developing world is illustrated by several recent events. Treatments for HIV infection are urgently needed in Africa, yet clinical trials of the anti-HIV drug tenofovir were halted in Cambodia, Cameroon and Nigeria because of claims that efforts to inform and involve local communities were inadequate ${ }^{9}$. And, in the face of famine, the Zambian government rejected food donated by the United States because it was genetically modified ${ }^{10}$. On a longer timescale, there was a large delay between the development of a vaccine against hepatitis B in the developed world and its widespread availability in the developing world.

At present, there is great interest in exploring such issues because of the unprecedented increase in financial resources for improving the health of individuals in the world's poorest countries. For example, since 2005, biomedical research projects focusing on the developing world have received US $\$ 450$ million from the Grand Challenges in Global Health initiative of the Bill \& Melinda Gates Foundation. With the increase in resources comes added responsibility to ensure that optimal improvements are made.
We set out to identify the factors, or forces, that affect the uptake of health-related biotechnology in the developing world. To do so, we interviewed 70 key experts from various sectors - academia, industry, civil society (voluntary and civic organizations) and government - in developing countries (see Supplementary Information for full details and breakdown)

These interviews allowed us to identify eight key areas that affect the development and adoption of health-related biotechnology in resource-poor regions. These areas can be categorized into four main forces: scientific, social (including ethical and cultural), financial and political. As a result, we were able to generate a model that can be used to assess the likelihood of success of health-related biotechnologies (see graphic, overleaf).

\section{The scientific question}

In terms of science, the participants in our study highlighted scientific capacity, infrastructure and collaboration as important.

Inadequate scientific capacity and infrastructure - for research, manufacturing and delivery - slow the development and adoption of health-related biotechnologies. Our interviewees emphasized that building the capacity for scientific research in the developing world - for example, improving training, facilities and equipment - enables researchers to investigate neglected diseases. But even for nations that have a strong research record, such as South Africa, the capacity for manufacturing remains a bottleneck. Local development and manufacturing, where possible, help local economies, making new technologies more sustainable and acceptable.

In the developing world, especially in rural areas, poor infrastructure also hampers the distribution of medicines. For example, understaffed health clinics need to cope with unreliable or prohibitively expensive transport. They often have no electricity or are plagued by power cuts. And they can lack potable water for administering pills.

Scientific collaboration can improve local scientific capacity, potentially enabling the developing world to contribute to the development of new technologies. But collaborations

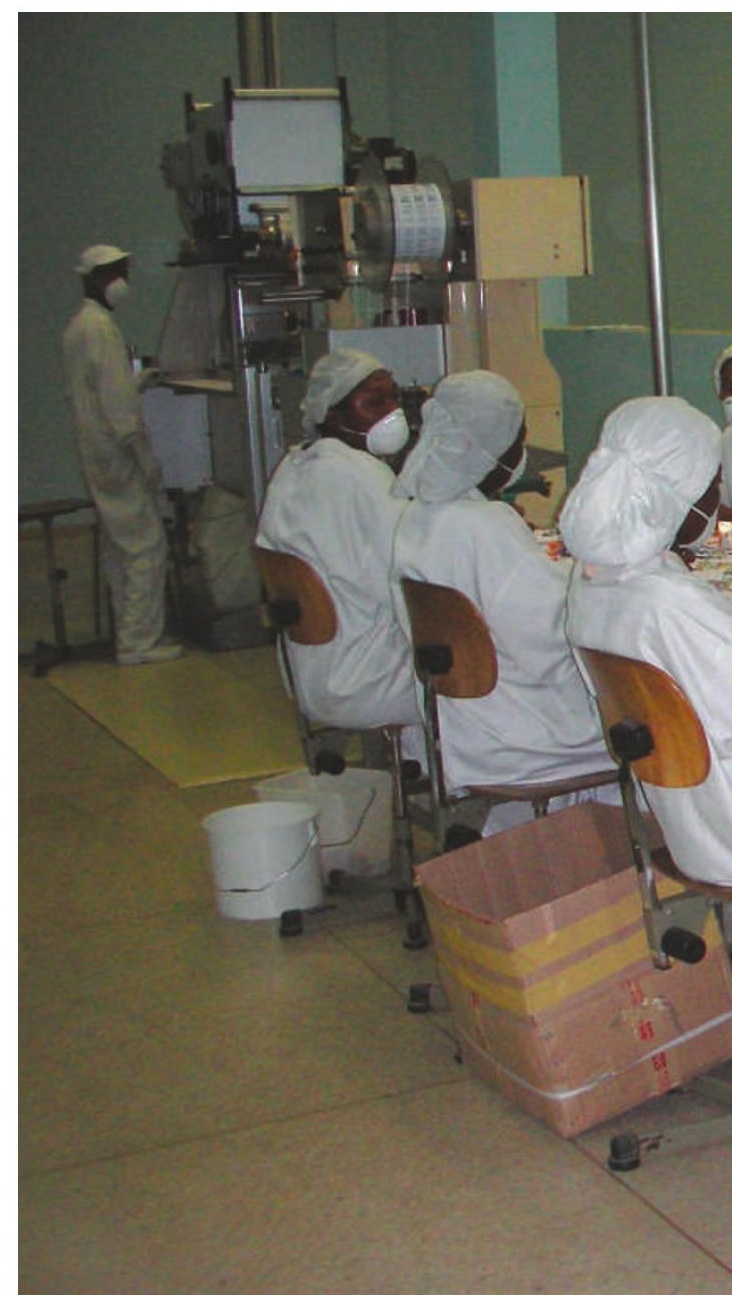

set up between scientists in the developing world and those in the developed world must be defined at the outset to avoid potential conflicts or exploitation.

One-third of all interviewees recommended classifying collaborations between scientists in the Northern Hemisphere and Southern Hemisphere as 'south-north' to remove any connotation of northern dominance. In addition, south-south collaborations hold promise for developing regionally relevant healthrelated biotechnologies and for creating sustainable benefits in the developing world. But care must be taken to avoid poaching scientific experts from other southern countries.

\section{Social issues}

When it comes to social issues, engaging the local community or general public and understanding the acceptability of products to the local culture seem to be key.

Engaging communities involves both authorization and consent. It depends on a legitimate and democratic leadership, and the acceptance of, and involvement in, new research strategies by the community. Our interviewees highlighted the need for early community engagement in any negotiations and deliberations, to help relationships between investigators and communities to be successful. Furthermore, they indicated that 


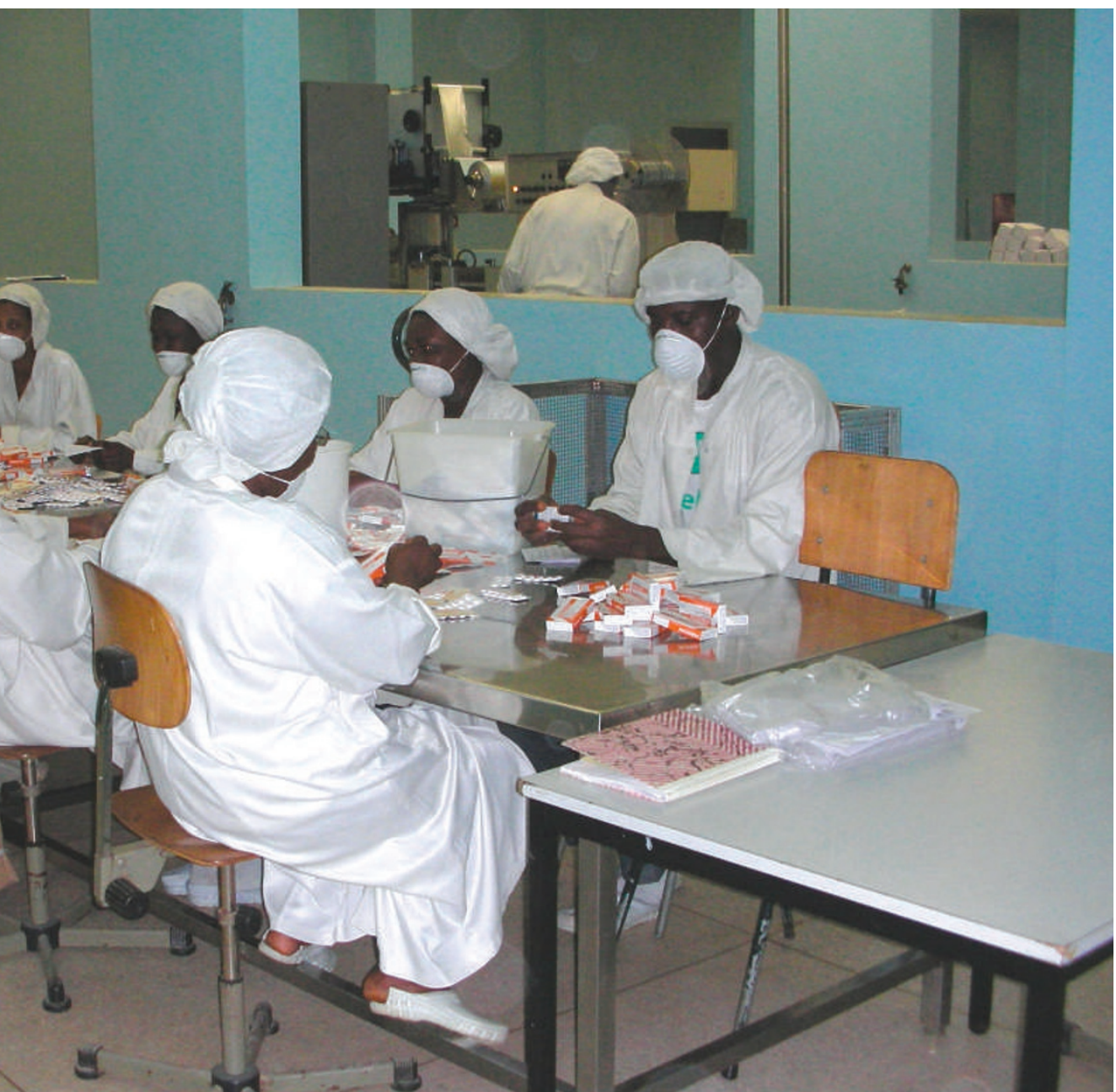

Act local: drugs made in Ghana are cheaper and more acceptable to the local community.

informed consent and community ownership, as well as access of participants to health care while enrolled in the trial and to successful drugs after the trial, are crucial to prevent abuse of the community.

Engaging the public helps to overcome the social barriers against new technologies such as nutritionally enhanced foods, intravaginal vaccines and genetic strategies to control mosquitoes. Information from radio, television and print media can help members of the public to evaluate their options. Similarly, educational campaigns led by religious leaders, political champions, tribal chiefs or teachers can enable people to make informed choices.

On the basis of previous vaccination and reproductive-health campaigns, the adoption of certain new technologies, including several being developed by the Grand Challenges in Global Health initiative, will require a well-designed strategy for engaging the public, gauging their views and concerns, and addressing these when implementing the intervention.

The cultural acceptability of new technologies must not be neglected, otherwise they will not reach the people who need them. To raise cultural awareness, researchers should allocate resources to the understanding of cultural diversity. This diversity is rooted in complex, interrelated cultural issues that stem from gender, religion, historical context, sexual practices and the use of contraception, and the absence of a culture of science in some countries. But as Musimbi Kanyoro, then general secretary of the World YWCA, said, culturally derived moral positions themselves have limits: "How can we, at this moment, let morality override mortality when we see the numbers of dead?"

\section{Financial considerations}

When it comes to finance, what is really meant is affordability and commercialization. New medicines developed as a result of advances in biotechnology must be affordable to people in developing countries (see page 176), and new drugs should be commercially viable in low-resource settings.

The commercialization of products for developing countries might entail financial risk. And entrepreneurs must take into account the limited funds available for production and the small profit margins in these regions. One way to boost commercialization is to manufacture drugs locally, making these drugs more affordable.

Increased affordability, in turn, results in access to larger markets and more opportunities

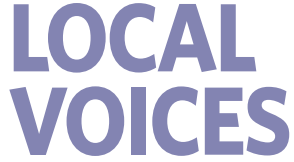

People working in developing countries offer their take on what affects the success of biotechnology in these regions.

"C You can't even begin to think about vaccines until you've succeeded in delivering electricity that will run refrigerators or have solar-powered refrigerators or something in the area to keep the vaccine cold." "

(6

What we want Western participation for is to bring innovation into thinking here - not to think for us. ")

\section{" You may end up with this fantastic, efficacious magic bullet, but if you don't understand some of the local culture and customs etcetera, then the uptake and acceptability are low.")}

\section{“ There's a big division between what the needs are in these countries and where drug development or other biotechnologies are actually located and shaped."}

\section{“ If a particular technology is very expensive, then the chance that it will be adopted and solve a particular problem is likely to be low.》”}


« The principle of justice demands that if a technology is found to be effective it should be made affordable to the population on whom it was experimented. $)$

\section{(C In typical African culture, men are always perceived as superior, and as a result, women do not have a voice."}

\section{‘c Researchers will continue to work hard to develop an efficacious product, but no product will reach the people who need it if the policy- makers won't support it. ")}

\section{" India provides an enormous cost advantage in process and clinical drug development. Multinational pharmaceutical companies have been forced to substantially reduce their prices.)}

\section{c Western scientists come with ready-made plans, and these don't necessarily answer local questions. ’’}

\section{" It comes back to political will and guts. Someone has to step up there, be the champion and say 'Come hell or high water, this is what we're going to do'. ")}

"There's a danger that much
of this research that is being
done on technology will result
in nothing because we have
not understood what the
community needs are."

for industry to balance profit and social benefit. An example of successful commercialization is India's first domestically produced and marketed recombinant vaccine against hepatitis $\mathrm{B}$. The innovative manufacturing processes used by Shantha Biotechnics, in Hyderabad, made this vaccine affordable to the Indian population $^{11}$. Another example is the vaccine against human rabies produced by Indian Immunologicals, also based in Hyderabad. The company developed a distribution network of refrigerated vehicles and franchise clinics to ensure that the vaccine reaches rural villages ${ }^{11}$.

Another crucial challenge for industry in the developing world is the weakness or absence of regulatory systems, which stifles innovation and patent registration and undermines the public's trust. Furthermore, our interviewees acknowledged that respect for intellectual property will be crucial if countries are to join the 'development ladder' (see page 174). But they emphasized that addressing global health crises will require ingenuity in devising commercialization strategies and guarding intellectual property.

For many interviewees, the issue of affordability was underpinned by considerations of fairness. Interviewees saw clear roles and responsibilities for providing affordable technologies to the poor on the part of the private sector, the government and international organizations. These involve differential pricing (supplying products to different markets at different prices) and single-buyer markets (for example, governments or the World
Health Organization), subsidies for vaccines and drugs, building up infrastructure and providing health care. The moral imperative to make products affordable is especially strong when considering those who participate in successful clinical trials but cannot afford to continue treatment when the trial ends. Overall, the main challenge in terms of finances is to balance affordability with incentives to innovate.

\section{Politics and policy}

The final area that affects the development and uptake of health-related biotechnology is politics: political will and the domestic policy environment. Our interviewees stressed that governments should include biotechnology in policies aimed at broadening national development and that these policies should span the sectors of medicine, health, development and trade.

Governments have the potential to fund the development and manufacture of drugs and to supply the single-buyer markets that make drugs affordable. Our interviewees called for governments in the developing world to follow the example of countries such as Brazil, China, Cuba, India, Rwanda, South Africa and Thailand, which have reaped the benefits of policies giving priority to science and technology. For example, the world's first vaccine against infection with Neisseria meningitidis serogroup B, which causes meningitis ${ }^{12}$, was produced in Cuba. Antigens manufactured in Cuba are also distributed to other developing countries: for

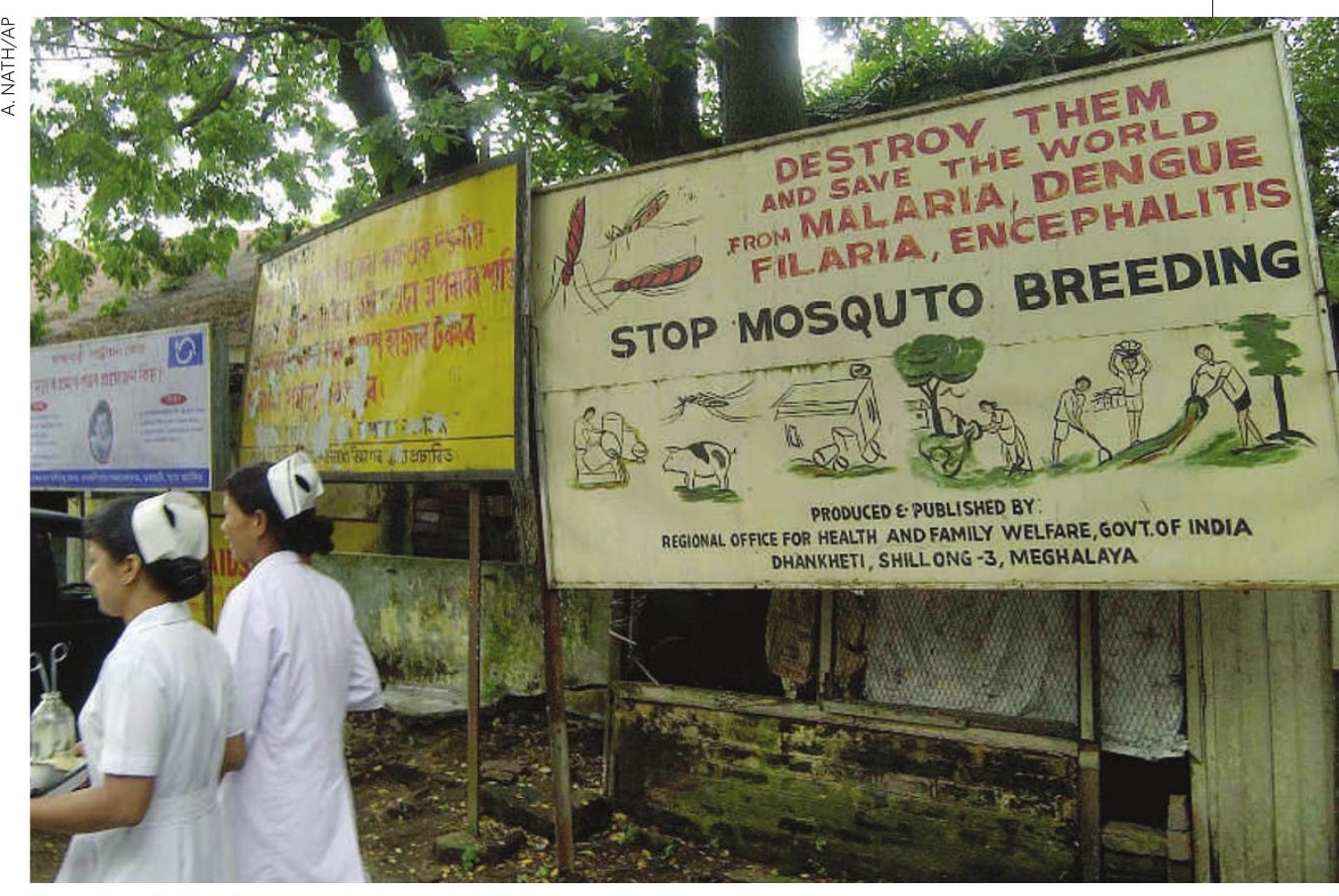

Engaging the local community is crucial to the success of new ideas. 


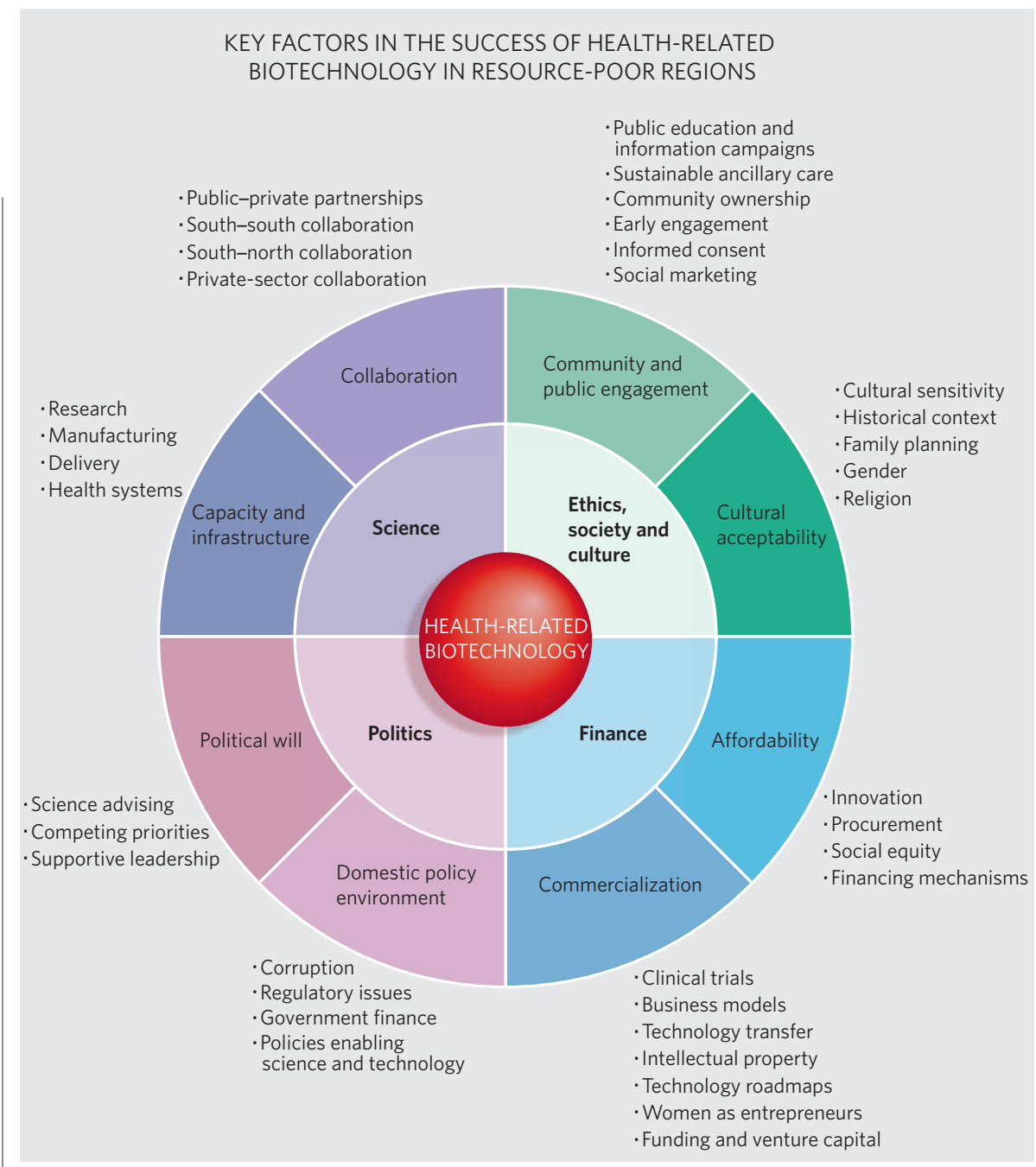

example, for the hepatitis B vaccine produced at South Africa's Biovac Institute in Wadeville.

Corruption, competing political priorities, inadequate public infrastructure for health care and anti-biotechnology lobbies can all block funding and access to new health products. Affordable hepatitis B vaccines, for example, fail to reach South Africans who need them, partly because public-health programmes are not politically expedient. Strong political champions, however, can forge progress. This has been shown by the New Partnership for Africa's Development, which has increased the visibility of science and innovation to African heads of state and has acknowledged the link between economic transformation and innovation in science and technology.

\section{A model for the future}

Several of those interviewed for our study stressed the importance of a broad approach to health-related biotechnology issues. For example, Adi Paterson, Group Executive of the South African government's Department of Science and Technology, urged all investigators to consider ethical factors in their analysis: "When a model is science-centric, it loses its ability to actually reflect early on the ethical question." bled a model that can be used to assess the potential success of particular health-related biotechnologies with respect to bottlenecks, regional variations and changes over time. (see graphic, above). In addition to the more obvious issues relating to science and finance, our model highlights that other issues - ethical, social, cultural and political - need to be addressed when seeking to improve health in the developing world.

It provides a framework for taking healthrelated biotechnology from the lab to the village. It offers a guide to structuring product and market readiness and to identifying factors that could impede the development of new products, such as vaccines, nutritionally enhanced foods, chemical and genetic strategies to control vectors, and diagnostics.

Using the model to assign priorities should streamline the development and adoption of emerging technologies. The model could, for example, be applied to the 2007 report Freedom to Innovate: Biotechnology in Africa's Development by the African Union and the New Partnership for Africa's Development, specifically to the 20-year comprehensive approach to fostering health-related biotechnology in the region. In this context, the model could
As a result of our interviews, we have assem- provide a blueprint for commercializing African health products.

We asked our study participants only about health-related biotechnology, so we cannot claim that the model applies to other types of technology, although it might resonate with some. We also acknowledge that the limited number of Latin American participants could diminish the scope of the model's application in that region, so the model might not apply in all developing-world contexts. However, key themes recurred in different regions, supporting the general relevance of the model. Some components of the model might seem simple and self-evident to experts, but this only affirms the validity of our empirically generated model.

Backed by the voices of experts in the developing world, the model enables the complex issue of biotechnology development to be systematically tackled, through examining the forces that affect health-related biotechnology in developing countries. The millions of deaths from preventable diseases every year demand clarity in interpreting and addressing these factors.

Peter A. Singer, Kathryn Berndtson, C. Shawn Tracy, Emma R. M. Cohen, Hassan Masum and Abdallah S. Daar are in the Program on Life Sciences, Ethics and Policy, at the McLaughlin-Rotman Centre for Global Health, Toronto, Canada. James V. Lavery is at the Centre for Research on Inner City Health and the Centre for Global Health Research, at St Michael's Hospital, Toronto, Canada.

1. Daar, A. S. et al. Nature Genet. 32, 229-232 (2002)

2. Morel, C. et al. Science 309, 401-404 (2005).

3. Maitland, C. Al Soc. 13, 341-356 (1999).

4. Geroski, P. A. Res. Pol. 29, 603-625 (2000)

5. Keller, W. J. Econ. Lit. XLII, 752-782 (2004).

6. Widdus, R. \& White, K. Combating Diseases Associated with Poverty: Financing Strategies for Product Development and the Potential Role of Public-Private Partnerships (Initiative on Public-Private Partnerships for Health, Global Forum for Health Research, Geneva, 2004)

7. Commission on Intellectual Property Rights, Innovation and Public Health. Public Health Innovation and Intellectual Property Rights (World Health Organization, Geneva, 2006).

8. Stoneman, P. \& Diederen, P. Econ. J. 104, 918-930 (1994)

9. Singh, J. A. \& Mills, E. J. PLoS Med. 2, e234 (2005).

10. Mitchell, P. Nature Biotechnol. 21, 6 (2003)

11. Frew, S. E. et al. Nature Biotechnol. 25, 403-417 (2007).

12. Thorsteinsdóttir, H. et al. Nature Biotechnol. 22, DC48-DC52 (2004)

Supplementary information is linked to the online version of this article at www.nature.com/nature

Acknowledgements We thank the study participants; the Ethical, Social and Cultural Program Team, especially A. Bhan and P. Tindana; the Grand Challenges in Global Health initiative staff for comments; and M. Keating for editorial input. This study was funded by a grant from the Bill \& Melinda Gates Foundation, through the Grand Challenges in Global Health initiative. 